\title{
An Implementation of Conjugate Gradient Methods for Estimating Polynomial Models
}

\author{
Osman O. O. Yousif \\ Department of Mathematics, \\ Faculty of Mathematical and \\ Computer Sciences, \\ University of Gezira, Sudan
}

\author{
Adam Hussein \\ Department of Statistics and \\ population studies, \\ Faculty of Economics and Social \\ Sciences, \\ Red Sea University, Sudan
}

\author{
Abdelrhman Abashar \\ Department of Basic Science, \\ Faculty of Engineering, \\ Red Sea University, Sudan
}

\begin{abstract}
Conjugate gradient (CG) methods are one of the most widely used methods for solving nonlinear unconstrained optimization problems, especially of large scale. That is, due to their simplicity and low memory requirement. To analyze the convergence properties of a CG method, it implemented into two line searches; exact and inexact. In this paper, given some data, some CG methods will be used to find a polynomial function that fitting the data. To show the efficiency, a comparison between CG methods and least square method will be done.
\end{abstract}

\section{Keywords}

Conjugate gradient methods; unconstrained optimization; least square; data fitting.

\section{INTRODUCTION}

To solve the unconstrained optimization problem

$$
\min f(x), x \in R^{n},
$$

where $f: R^{n} \rightarrow R$ is continuously differentiable and it's gradient at a point $x_{k}$ is denoted by $g_{k}$ or $\nabla f\left(x_{k}\right)$, the CG methods use the iterative formula

$x_{k+1}=x_{k}+\alpha_{k} d_{k}$,

where $\alpha_{k}$ is step size which can be obtained by the exact line search

$$
f\left(x_{k}+\alpha_{k} d_{k}\right)=\min _{\alpha \geq 0} f\left(x_{k}+\alpha d_{k}\right)
$$

which simply means the orthogonality condition

$g_{k}^{T} d_{k-1}=0$,

is hold. Also, the step size can be computed by inexact line search methods such as the strong Wolfe

$$
\begin{gathered}
f\left(x_{k}+\alpha_{k} d_{k}\right) \leq f\left(x_{k}\right)+\delta \alpha_{k} g_{k}^{T} d_{k} \\
\left|g\left(x_{k}+\alpha_{k} d_{k}\right)^{T} d_{k}\right| \leq \sigma\left|g_{k}^{T} d_{k}\right|,
\end{gathered}
$$

where $0<\delta<\sigma<1$. In (1.2), $x_{k}$ is the current iterate and $d_{k}$ is the search direction that defined by

$d_{k}= \begin{cases}-g_{k}, & \text { if } k=0 \\ -g_{k}+\beta_{k} d_{k-1}, & \text { if } k \geq 1,\end{cases}$

where $\beta_{k}$ is a parameter that whose different forms determine different conjugate gradient methods. Well known formulas for $\beta_{k}$ are the Hestenes-Stiefel (HS) [6], Fletcher-Reeves (FR) [10], Polak-Ribière-Polyak (PRP) [2, 3], conjugate descent (CD) [9], Liu-Storey (LS) [11], and Dai-Yuan (DY) [12] formulas. For more formulas for the coefficient $\beta_{k}$ see $[4,5$, $9,13,14,15$, and 16].

In this paper, a new algorithm that uses CG methods to fit some data by a polynomial of degree $n$ will be presented in the next section. In Section 3, a numerical experiment that shows the efficiency of the new algorithm will be done. A conclusion will be presented in Section 4.

\section{THE NEW ALGORITHM}

It is well known that the method of least squares is a mathematical procedure for finding the best fitting-curve to a given set of points by minimizing the sum of the squares [1]. It can be used to fit the data $\left(x_{i}, y_{i}\right), i=1,2, \ldots, n$ using a polynomial of degree $n$, that is

$y=a_{0}+a_{1} x+\cdots a_{n} x^{n}$.

When the data $\left(x_{i}, y_{i}\right), i=1,2, \ldots, n$ substituted in in the polynomial above, errors $\epsilon_{i}$ occurs, that is,

$y_{i}=a_{0}+a_{1} x_{i}+\cdots a_{n} x_{i}^{n}+\epsilon_{i}, \quad i=1,2, \ldots, n$.

Therefore, the sum of the square of errors is given by

$$
\begin{aligned}
E(a) & =\sum_{i=1}^{n}\left(y_{i}-a_{0}+a_{1} x_{i}+\cdots a_{n} x_{i}^{n}\right)^{2} \\
& =\sum_{i=1}^{n}\left(y_{i}-a^{T} x_{i}\right)^{2}
\end{aligned}
$$

where

$$
\begin{aligned}
& a^{T}=\left[\begin{array}{llll}
a_{0} & a_{1} & \ldots & a_{n}
\end{array}\right] \text { and } x_{i}=\left[\begin{array}{llll}
1 & x_{i} & \ldots & x_{i}^{n}
\end{array}\right]^{T}, \\
& i=1,2, \ldots, n .
\end{aligned}
$$

Equation (2.1) is a continuously differentiable function in the variable $a$ and its gradient is given by

$$
\nabla E(a)=\left[\begin{array}{llll}
\frac{\partial E}{\partial a_{0}} & \frac{\partial E}{\partial a_{1}} & \ldots & \frac{\partial E}{\partial a_{n}}
\end{array}\right]^{T}
$$

where

$$
\frac{\partial E}{\partial a_{j}}=\sum_{i=1}^{n} 2 x_{i}^{j}\left(y_{i}-a^{T} x_{i}\right), \quad j=0,1,2, \ldots, n .
$$

To find approximation values of the coefficients $a$, the least square minimizes the sum of the square of errors, that is solve $\min E(a)$ 
The problem (2.3) is a nonlinear unconstrained optimization in the variable $a$ which can be solved using CG methods.

The following algorithm uses the CG techniques to solve problem (2.3) provides the line search is exact.

Algorithm 2.1: An algorithm for solving problem (2.3)

Step 1: $\quad$ Choose $x_{0} \in R^{n}, \varepsilon \geq 0$.

Step 2: $\quad$ Compute the gradient (2.2) at $x_{0}$

Step 3: $\quad$ Set $\mathrm{k}=0$ and $d_{\mathrm{k}}=-g_{\mathrm{k}}$

Step 4: $\quad$ if $\left\|g_{0}\right\| \leq \varepsilon$, then stop.

Step 5: $\quad$ Compute $\alpha_{\mathrm{k}}$ by exact line search (1.3).

Step 6: $\quad$ Set $a_{k+1}=a_{k}+\alpha_{k} d_{k}$

Step 7: $\quad$ Compute the gradient (2.2) at $a_{k+1}$

Step 8: $\quad$ if $\left\|g_{k+1}\right\|<\varepsilon$, then stop.

Step 9: $\quad$ Compute the parameter $\beta_{k}$ and generates $d_{k}$ by (1.4).

Step 10: $\quad$ Set $k=k+1$ go to Step 5 .

\section{NUMERICAL EXPERIMENT}

In this section, to show the efficiency of Algorithm 2.1, it applied on some data obtained from [7]. This data is listed in Table 1.

Table 1: Unemployment rate in Terengganu from 2000 to 2015

\begin{tabular}{ccc}
\hline No. & Years & Unemployment Rate \\
\hline 1 & 2000 & 3.0 \\
2 & 2001 & 2.7 \\
3 & 2002 & 3.2 \\
4 & 2003 & 2.9 \\
5 & 2004 & 3.2 \\
6 & 2005 & 3.1 \\
7 & 2006 & 3.6 \\
8 & 2007 & 2.6 \\
9 & 2008 & 3.4 \\
10 & 2009 & 3.8 \\
11 & 2010 & 3.7 \\
12 & 2011 & 3.2 \\
13 & 2012 & 3.0 \\
14 & 2013 & 3.4 \\
15 & 2014 & 4.2 \\
16 & 2015 & 4.0 \\
\hline
\end{tabular}

The data in Table 1 can be plotted using MATLAB in Fig. 1.

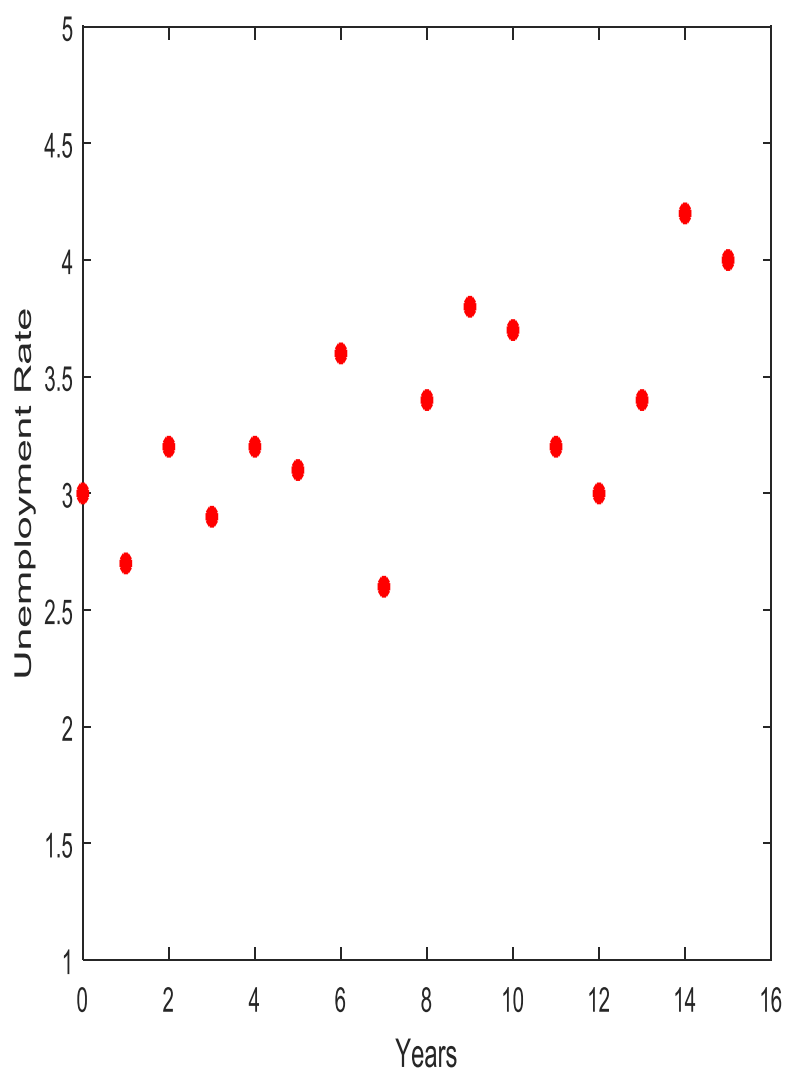

Fig. 1: Plotting of unemployment versus years

Using the least square calculations, it can be found that the linear, quadratic, and cubic polynomials that fitting the data in Table 1 are

$y=2.8493+0.0618 x$,

$a=\left[\begin{array}{ll}2.8493 & 0.0618\end{array}\right]^{T}$,

$y=2.9547+.0166 x+0.0030 x^{2}$,

$a=\left[\begin{array}{lll}2.9547 & 0.0166 & 0.0030\end{array}\right]^{T}$

$y=2.8233+0.1424 x+-0.0186 x^{2}+0.001 x^{3}$,

$a=\left[\begin{array}{llll}2.8233 & 0.1424 & -0.0186 & 0.001\end{array}\right]^{T}$

respectively.

To solve (2.3), a MATLAB coded program for Algorithm 2.1, with different values of the parameter $\beta_{k}$, hence different CG methods, namely, FR, PRP, and the new method [8] whose parameter given by

$$
\beta_{k}^{O R}= \begin{cases}\frac{g_{k}^{T} g_{k}-\left|g_{k}^{T} d_{k-1}\right|}{d_{k-1}^{T} d_{k-1}}, & \text { if } g_{k}^{T} g_{k} \geq\left|g_{k}^{T} d_{k-1}\right| \\ 0, & \text { otherwise, }\end{cases}
$$

and with different initial points, has been run with stopping criterion $\left\|g_{k}\right\|<10^{-6}$. The numerical results are in Table 2 . 
Table 2: Implementation of FR, PRP, and OR methods for solving problem (2.3)

\begin{tabular}{|c|c|c|c|c|}
\hline Poly. & Initial point & FR & PRP & OR \\
\hline Linear & $(0,0)$ & $(2.8493,0.0618)$ & $(2.8493,0.0618)$ & $(2.8493,0.0618)$ \\
& $(10,10)$ & $(2.8493,0.0618)$ & $(2.8493,0.0618)$ & $(2.8493,0.0618)$ \\
& $(50,50)$ & $(2.8493,0.0618)$ & $(2.8493,0.0618)$ & $(2.8493,0.0618)$ \\
& $(-100,-100)$ & $(2.8493,0.0618)$ & $(2.8493,0.0618)$ & $(2.9547,0.0166,0.0030)$ \\
\hline Quad. & $(0,0,0)$ & $(2.9547,0.0166,0.0030)$ & $(2.9547,0.0166,0.0030)$ & $(2.9547,0.0166,0.0030)$ \\
& $(20,20,20)$ & $(2.9547,0.0166,0.0030)$ & $(2.9547,0.0166,0.0030)$ & $(2.9547,0.0166,0.0030)$ \\
& $(-10,-10,-10)$ & $(2.9547,0.0166,0.0030)$ & $(2.9547,0.0166,0.0030)$ & $(2.9547,0.0166,0.0030)$ \\
& $(-40,-40,-40)$ & $(2.9547,0.0166,0.0030)$ & $(2.9547,0.0166,0.0030)$ & $(2.8233,0.1424,-.0186,0.001)$ \\
& $(0,0,0,0)$ & $(2.8233,0.1424,-.0186,0.001)$ & $(2.8233,0.1424,-0.0186,0.0010)$ & $(2.8233,0.1424,-.0186,0.001)$ \\
& $(5,5,5,5)$ & $(2.8233,0.1424,-0.0186,0.0010)$ & $(2.8233,0.1424,-0.0186,0.0010)$ & $(2.8233,0.1424,-.0186,0.001)$ \\
& $(-1,-1,-1,-1)$ & $(2.8233,0.1424,-0.0186,0.0010)$ & $(2.8233,0.1424,-0.0186,0.0010)$ & $(2.8233,0.1424,-.0186,0.001)$ \\
& $(1,10,1,10)$ & $(2.8233,0.1424,-0.0186,0.0010)$ & $(2.8233,0.1424,-0.0186,0.0010)$ & \\
\hline
\end{tabular}

Obviously, from Table 2, FR, PRP, and OR solve problem (2.3) and the results coincide with (3.1), (3.2), and (3.3). Therefore, they give a good fitting for the data in Table 1 as in Figure 2 .

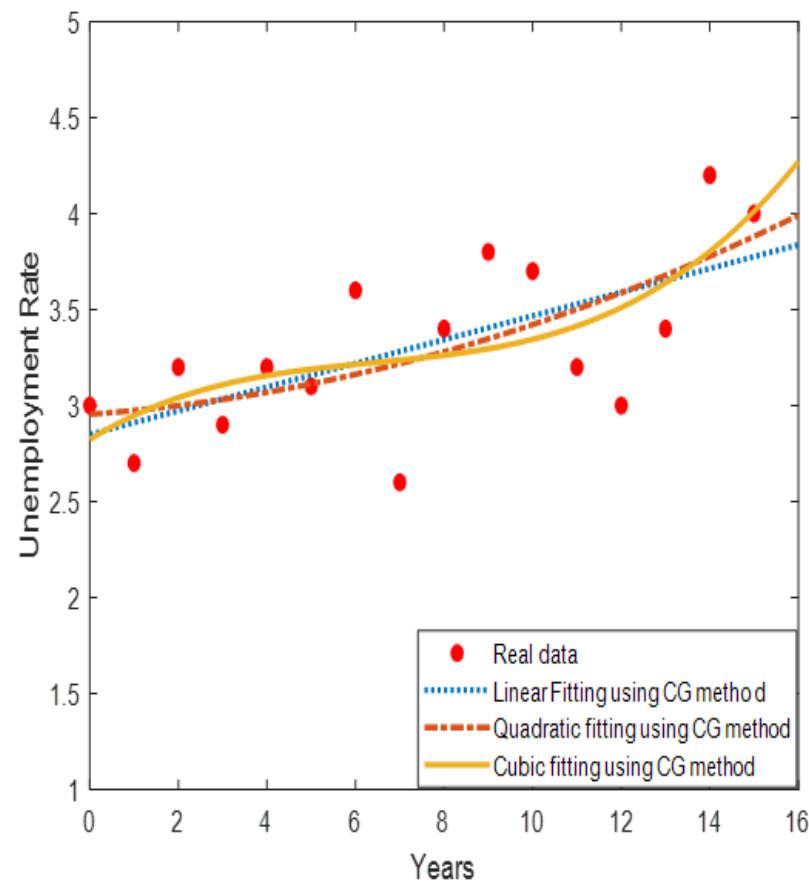

Figure 2: Curve fitting using CG methods

\section{CONCLUSION}

In this paper, based on some data, a conjugate gradient algorithm with exact line search has been applied to construct linear, quadratic, and cubic polynomials that fitting the data. To show the efficiency of conjugate gradient methods, a numerical experiment has been done. It has been shown that FR, PRP, and the new OR methods give the same results when they used for fitting data by polynomials.

\section{REFERENCES}

[1] Abdelwab Kharab and Ronald B. Guenther (2012), An introduction to numerical methods A matlab approach, CRC press.

[2] B. T. Polyak, The conjugate gradient method in extremem problems, USSR Comp. Math. Math. Phys., 9 (1969), pp. 94-112.

[3] E. Polak, Optimization: Algorithms and Consistent Approximations, vol. 124 of Applied Mathematical Sciences, Springer, New York, NY, USA, 1997.

[4] G. Yuan and X. Lu, "A modified PRP conjugate gradient method", Annals of Operations Research, vol. 166, pp. 73-90, 2009.

[5] L. Zhang, An improved Wei-Yao-Liu nonlinear conjugate gradient method for optimization computation, Appl. Math. Comput. 215 (2009), pp. 2269-2274.

[6] M. R. Hestenes and E. L. Stiefel, Methods of conjugate gradients for solving linear systems, J. Research Nat. Bur. Standards. 49 (1952), pp. 409-436.

[7] Nur Syarafina, M., Mustafa, M., Rivaie, M., Nur Hamizah, A., Norhaslinda Z., Syazni S., Estimating the unemployment rate using least square and conjugate gradient methods. International Journal of Engineering and Technology, 7(2.15), (2018) 94- 97.

[8] Osman, O.,Mamat, M., Abdelrhaman, A., Rivaie, M. (2014).The global convergence properties of a conjugate gradient methods. American Institute of Physics Conference Proceeding, 1602 (286), 286-295.

[9] R. Fletcher, Practical Method of Optimization, Vol. 1, Unconstrained Optimization, John Wiley \& Sons, New York, 1987.

[10] R. Fletcher and C. Reeves, Function minimization by conjugate gradients, Comput. J., 7 (1964), pp. 149-154.

[11] Y. Liu, C. Storey, Efficient generalized conjugate gradient algorithms. Part 1: Theory, J. Optimiz. Theory Appl. 69 (1992), pp. 129-137. 
International Journal of Computer Applications (0975 - 8887)

Volume 178 - No. 51, September 2019

[12] Y. H. Dai, Y. Yuan, A nonlinear conjugate gradient method with a strong global convergence property, SIAM J. Optim. 10 (2000), pp. 177-182.

[13] W. W. Hager and H. Zhang, "A new conjugate gradient method with guaranteed descent and an efficient line search," SIAM Journal on Optimization, vol. 16, no. 1, pp. 170-192, 2005.

[14] Y. Dai, "A nonmonotone conjugate gradient algorithm for unconstrained optimization," Journal of Systems
Science and Complexity, vol. 15, no. 2, pp. 139-145, 2002.

[15] Z. Wei, G. Li, and L. Qi, "New nonlinear conjugate gradient formulas for large-scale unconstrained optimization problems," Applied Mathematics and Computation, vol. 179, no. 2, pp. 407-430, 2006.

[16] Z. Wei, S. Yao, and L. Liu, "The convergence properties of some new conjugate gradient methods," Applied Mathematics and Computation, vol. 183, no. 2, pp. 13411350, 2006 